\title{
Vertical orientation of solvent cast nanofilled PS- $b$-PEO block copolymer thin films at high nanoparticle loading
} \author{
Abul F. Huq, Manish Kulkarni, ${ }^{1,2}$ Arvind Modi, Detlef-M. Smilgies, ${ }^{3}$
} Abdullah M. Al-Enizi ${ }^{4}$, Ahmed Elzatahry ${ }^{4+}$, Dharmaraj Raghavan ${ }^{5}$ and Alamgir Karim

1 Department of Polymer Engineering, the University of Akron, Akron, OH 44304, United States

Current address: DST Unit of Nanosciences, Department of Chemical Engineering, Indian Institute of Technology, Kanpur, UP -208016, India

3

Cornell High Energy Synchrotron Source (CHESS), 161 Synchrotron Drive, Wilson

Laboratory, Ithaca, NY 14853, United States

${ }^{4}$ Chemistry Department, Faculty of Science, King Saud University, PO Box 2455, Riyadh 11451, Saudi Arabia

${ }^{+}$Permanent Address: Polymer Materials Research Department, Advanced Technology and New Materials Research Institute, City of Scientific Research and Technology Applications, New Borg El-Arab City Alexandria 21934, Egypt

${ }^{5}$ Chemistry Department, Howard University, Washington D.C.20059

*alamgir@uakron.edu

KEYWORDS: mixed solvent, block copolymer, Hansen solubility parameter, nanoparticle dispersion

ABSTRACT: We demonstrate selective dispersion of photosensitive electron acceptor phenylC61-butyric acid methyl ester (PCBM) in cylinder forming polystyrene- $b$-poly (ethylene oxide) (PS- $b$-PEO) block copolymer (BCP). Neat PS- $b$-PEO is structurally robust to forming vertically oriented nanoscale morphology of PEO cylinders in the PS matrix directly after solvent casting. Varying amounts of PCBM nanoparticles were mixed with PS- $b$-PEO BCP in solvent mixtures and films were spin cast on UV/ozone treated clean silicon wafers. We studied in detail the BCP morphology orientation effect of different solvents and solvent mixtures for varying PCBM loading $\left(\phi_{\mathrm{PCBM}}\right)$ in the films, range from 0-50 weight percent relative to the BCP. The key accomplishments of this work include vertical orientation control even at large concentration of PCBM $\sim 30 \%(\mathrm{w} / \mathrm{w})$ particles relative to polymer mass and preferential segregation of PCBM in PS block via mixed solvent strategy which otherwise is difficult to realize due to segregation of PCBM on polar substrate. We explain preferential dispersion of particles in films by demarcating limits in terms of critical Hansen solubility parameter. This value is close to the percolation threshold of PCBM, so a conducting polymer matrix can be expected, especially when it is partitioned preferentially in the matrix PS domain. To fully quantify the nanofilled PS-PEO morphology, for a range of casting solvents, solvent mixture compositions and 
$\phi_{\mathrm{PCBM}}$, we measured changes in PEO cylinder diameter, cylinder center-to-center distance with PCBM incorporation into PS matrix, change of film thickness with overall PCBM incorporation and root mean square roughness of the film surface. Notably, the film thickness increased as the PCBM content in film was increased in the casting solvent mixtures, for same processing condition, so that domain swelling by nanoparticles was self-adjusting with increase of film thickness.

\section{Introduction}

Due to chemical incompatibility of the constituent polymer blocks in a block-copolymer (BCP), it can self-assemble in an ordered fashion into various morphologies with domain sizes as small as a few nanometers in thin films. ${ }^{1-5}$ Domain size, shape and structure can be exquisitely controlled by the constituent block chemistry and relative interaction. This enables potential applications of such systems in functional electronic devices such as solar cells, high-density magnetic storage, capacitors, etc. ${ }^{6-8}$ Darling et al. ${ }^{9}$ suggested that cylinder forming block copolymer thin films, where the two different domain phases consist of electron donors and acceptors, can be used as model systems for efficient charge separation and transportation in ordered bulk heterojunction solar cells. In this regard, regular patterns of block copolymers can also be used as partitioning templates for electron accepting nanoparticles in ordered arrays. ${ }^{10-17}$ Incorporation of additives such as functional nanoparticles into the block copolymer can modify the overall film property such as its dielectric constant for high energy density block copolymer capacitors. Cooperative and controllable interaction of block copolymer and nanoparticle often requires meticulous engineering and proper choices of nanoparticles and block copolymers. ${ }^{18-26}$ Hence, selective segregation and functional tunability offers a significant challenge.

Dispersion of nanoparticles into the BCP films may lead to some undesirable changes however for certain applications. For example, Zhang et al. ${ }^{38}$ observed that even at lowloading of AuNPs in PS-b-PMMA films $(\sim 2-3 \% \mathrm{w} / \mathrm{w})$, the PMMA cylinders oriented horizontally with respect to the substrate as opposed to vertically oriented cylinders observed in the neat BCP films. Further, the orientation parameter decreased with increase in particle loading to a mostly parallel cylinder state. Such a phenomena may be undesirable for certain applications such as membranes requiring vertical orientation of cylinders, and poses a challenge to nano-technology applications of BCP films with functionally loaded nanoparticles.

In this article we introduce a novel and facile mixed solvent strategy to (a) preferentially incorporate nanoparticles overall into the systems, or as needed, into a selected block copolymer phase and (b) control orientation of cylinders even at high loading of nanoparticles. This technique does not require modification of the polymer or nanoparticles, which are often undesirable and may influence the intended functionally of the composite structure. One of the well-studied block copolymer systems is polystyrene- $b$-poly(ethylene oxide) (PS- $b$-PEO) due to 
its unique combination of a hydrophobic and a hydrophilic block. Notably, films of PS- $b$-PEO cast from solvent can readily produce robust vertically ordered cylindrical morphology ${ }^{6,19}$, potentially important for nanotechnology applications such as efficient membranes, capacitors and solar cells. In this paper, we critically examine various aspects related to the synergy of PS$b$-PEO block copolymer self-assembly morphology when blended with an electron accepting nanoparticle, phenyl-C61-butyric acid methyl ester (PCBM), commonly used for polymer solar cells or modifying dielectric properties. Atomic force microscope (AFM) images are analyzed to examine several key morphological aspects of solvent cast PS- $b$-PEO block copolymer thin films filled with PCBM up to significantly high loading levels from 0 to $50 \mathrm{wt} \%$. In this regard, while we deal with a model BCP system, loading of other conjugated block copolymer films such as with P3HT as a block with added electron accepting nanoparticles may be particularly relevant for solar cell applications at $40-50 \mathrm{wt}$. \% loading levels. ${ }^{27}$

Previous studies have reported that the relative radius of gyration of polymer versus the nanoparticle can affect dispersion and selectivity of nanoparticle into block copolymers. ${ }^{25,26}$ The radius of gyration, $R_{g}$ of a polymer can be calculated for an unperturbed linear chain using the formula $R_{g}{ }^{2}=h 20 / 6$, where 020 is mean square of end-to-end distance of an unperturbed polymer chain. Values of $20 / M$ for poly(ethylene oxide) and polystyrene are 0.8 and 0.43 2 $2 \mathrm{~mol} / \mathrm{g}$ respectively ${ }^{28}$, where $M$ is the molecular weight in $\mathrm{g} / \mathrm{mol}$. Consequently, for poly(ethylene oxide) with $M=6,500 \mathrm{~g} / \mathrm{mol}$ the value of $R_{g E}$ is 29.4 and for polystyrene with $M=20,000 \mathrm{~g} / \mathrm{mol}$ the value of $R_{g S}$ is 37.9 a. The $R_{g}$ of the PS- $b$-PEO block copolymer can be calculated by ${ }^{4} R_{g b}{ }^{2}=$ $R_{g E}{ }^{2}+R_{g S}{ }^{2}$ which give an $R_{g b}$ value of 48 ?. Hence, the radius of gyration of the PCBM nanoparticle $\sim 1.06 \mathrm{~nm}(10.6$ ]), is much smaller compared to the radius of gyration of either of the blocks individually. In such case, the enthalpic contribution to the dispersion process due to creation of polymer-nanoparticle interface is less significant than the particle translational entropy. ${ }^{25}$

Transmission electron microscopy (TEM) measurements do not have sufficient contrast required to distinguish the PCBM molecules from either of the block copolymer components, and small angle neutron scattering (SANS) of multilayered stacked films would be potentially required to measure changes in molecular dimensions of the block copolymer, which is outside the scope of these studies. Our study shows that changes in molecular dimensions due to PCBM incorporation are equally reflected in changes in cylinder domain dimensions and inter-domain spacing. During the spin coating process the fast adsorption kinetics of the small molecule, PCBM, as compared to that of a slowly ordering macromolecular block copolymer, PS- $b$-PEO, results in its segregation at the substrate interface of the thin film. However, in this research utilizing a proper pair of mixed casting solvent in appropriate composition, we show that PCBM can be kinetically trapped in the PS phase. PCBM in excess of what can be incorporated through these molecular level changes of block copolymer domains are accommodated by changes in block copolymer film thickness or by locating them outside of the film structure at the film 
substrate interface. Specifically, the contribution to changes of in-plane dimensions affect the cylinder diameter and cylinder-to-cylinder spacing, while PCBM amount in excess of what can be accommodated in increase of in-plane dimensions are expended as out of plane or increase of film thickness or as aggregates at top and bottom of film depending on solvent casting conditions. Thus, this approach leads to formation of self-adjusting nanocomposite ordered block copolymer thin film system, with the ability to accommodate high nano-filler loading levels without losing basic block copolymer ordered microstructure. In the present study, we rely upon atomic force microscope (AFM) and grazing-incidence small-angle X-ray scattering (GISAXS) to elucidate the morphology of the film structure and changes with introduction of the PCBM nanoparticles. These include measurements of changes in cylinder diameter, center-to-center distance of two cylinders, film roughness and fast Fourier transform (FFT) for analysis of long range order. Film thickness, obtained through AFM scratch tests, determined how nanoparticles contributed to in-plane dimensions versus out of plane thickness. These results are potentially important to BCP films with functionally loaded nanoparticles at high concentration for inducing property changes such as plasmonics etc.

\section{Experimental}

PS- $b$-PEO (Number average molecular weight, $\mathrm{M}_{\mathrm{n}}=20 \mathrm{k}-b-6.5 \mathrm{k}$ ) with polydispersity index 1.06 was purchased from Polymer Source Inc. However, in spin coated thin films aged for $>24 \mathrm{~h}$, it was observed by optical microscope that the as-purchased polymer formed crystalline regions. We assume that this is due to crystallization of a PEO homopolymer impurity (glass transition temperature, $T_{g}$, of PEO is $-41^{\circ} \mathrm{C}$ ). In order to remove such impurities, a $25 \%(\mathrm{w} / \mathrm{w})$ PS- $b$-PEO polymer solution in toluene was prepared. Subsequently, methanol was added carefully and slowly into this solution so that the block copolymer precipitated out and the methanol-soluble PEO homopolymer remained in the solvent. Then the resultant PEO homopolymer rich solution was carefully taken out using micropipette from the top of the solution. Once most of the solution was removed, the precipitated PS- $b$-PEO block polymer was dried at $60^{\circ} \mathrm{C}$ for twelve hours under vacuum. The process was repeated until two consecutive weights of the residual polymer became similar, showing no further PEO homopolymer remained in the precipitate. 99.5\% purity PCBM from nano-C Inc. was used as purchased. In this work, (100) oriented p-type silicon wafer from Silicon Quest International, Inc., was rinsed with toluene and chloroform in sequence, and subsequently UVO treated for 30 min before being used as a substrate. Samples were prepared on these substrates by spin casting at $1000 \mathrm{rpm}$ from $0.90 \%(\mathrm{w} / \mathrm{w})$ purified PS- $b$-PEO dissolved in toluene. We tuned concentrations, such that thickness of all pure PS- $b$-PEO block copolymers cast from different solvents and solvent mixtures were around $50 \mathrm{~nm}$. PS- $b$-PEO concentrations was observed to vary for different mixed solvents so that thickness of the films remained close to $50 \mathrm{~nm}$.

The topography of the films was studied utilizing a Bruker Corporation's "Dimension Icon" AFM, operated in standard tapping mode. High resolution TEM images were taken 
utilizing Japan Electron Optics Laboratory (JEOL)'s model JSM-1230, transmission electron microscope. GISAXS experiments were performed at the D1 beam line of the Cornell High Energy Synchrotron Source (CHESS) and at the X9 beam line of the National Synchrotron Light Source (NSLS) at Brookhaven National Laboratory. The film internal morphology was studied utilizing GISAXS slightly above the critical angle of the film $\left(0.12^{\circ}\right)$ at the first waveguide resonance value. In this way, integrated information over the entire film, not just the surface, can be obtained with enhanced scattering. Grazing-incidence wide-angle X-ray scattering (GIWAXS) experiments were done at the D1 beam line of the Cornell High Energy Synchrotron Source (CHESS).

NanoScope Analysis, version v730R1sr2 of Bruker Corporation was employed to examine AFM data. Utilizing this software, the film roughness and first Fourier transform (FFT) of the in-plane surface ordering of block copolymer domains were directly obtained. Cylinder diameters were obtained by analyzing AFM phase images using ImageJ of National Institute of Health. For this purpose an image was first converted to 8-bit gray scale followed by applying "thresholding" function. Lower and upper limits for diameter cut offs were $11 \mathrm{~nm}$ and $25 \mathrm{~nm}$ and circularity, defined by $4 \pi \times$ Area/Perimeter ${ }^{2}$, range for acceptable diameter was between 0.5 and 1. Finally, OriginPro9.0 was used for statistical analysis of the cylinder size distribution data. Film thickness was obtained through AFM scratch test analyzed by NanoScope Analysis step function. Center-to-center distance of two cylinders, $l$, was obtained using the $q$ value at the

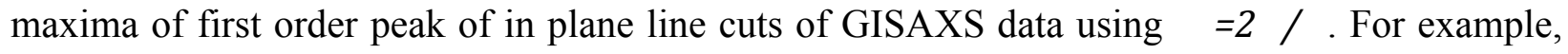
center-to-center distance of pure PS- $b$-PEO block copolymer obtained from GISAXS is 31.32 $\mathrm{nm}$ in agreement with AFM data. For pure block copolymer, cylinder diameter can also be

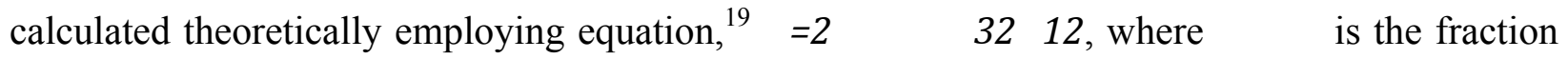
of PEO in block copolymer. Thus cylinder diameter obtained from above equation is $16.3 \mathrm{~nm}$, while the experimental value is $17.9 \mathrm{~nm}$. Fast precipitation of PEO due to its low solubility in toluene during rapid drying under fast spin casting process may be the reason behind slightly larger diameter of PEO than the calculated value.

\section{Results}

\section{Single Solvent Cast Ordered Nanofilled BCP Film Morphology}

We first examine the ordering of PCBM filled PS- $b$-PEO films cast from pure solvent, toluene. Even though PS is preferentially soluble in toluene as compared to PEO, the block copolymer at low concentration is not expected to form micelles. ${ }^{6}$. Figure 1a is a series of AFM height and phase images for a range of PCBM loading levels in PS- $b$-PEO films. It shows that for all levels of PCBM loadings in the PS- $b$-PEO films, the PEO domains forms vertical cylinders. Such robust structure is attributed due to the fact that PS (surface energy $\left.40.7 \mathrm{~mJ} / \mathrm{m}^{2}\right)^{29}$ 


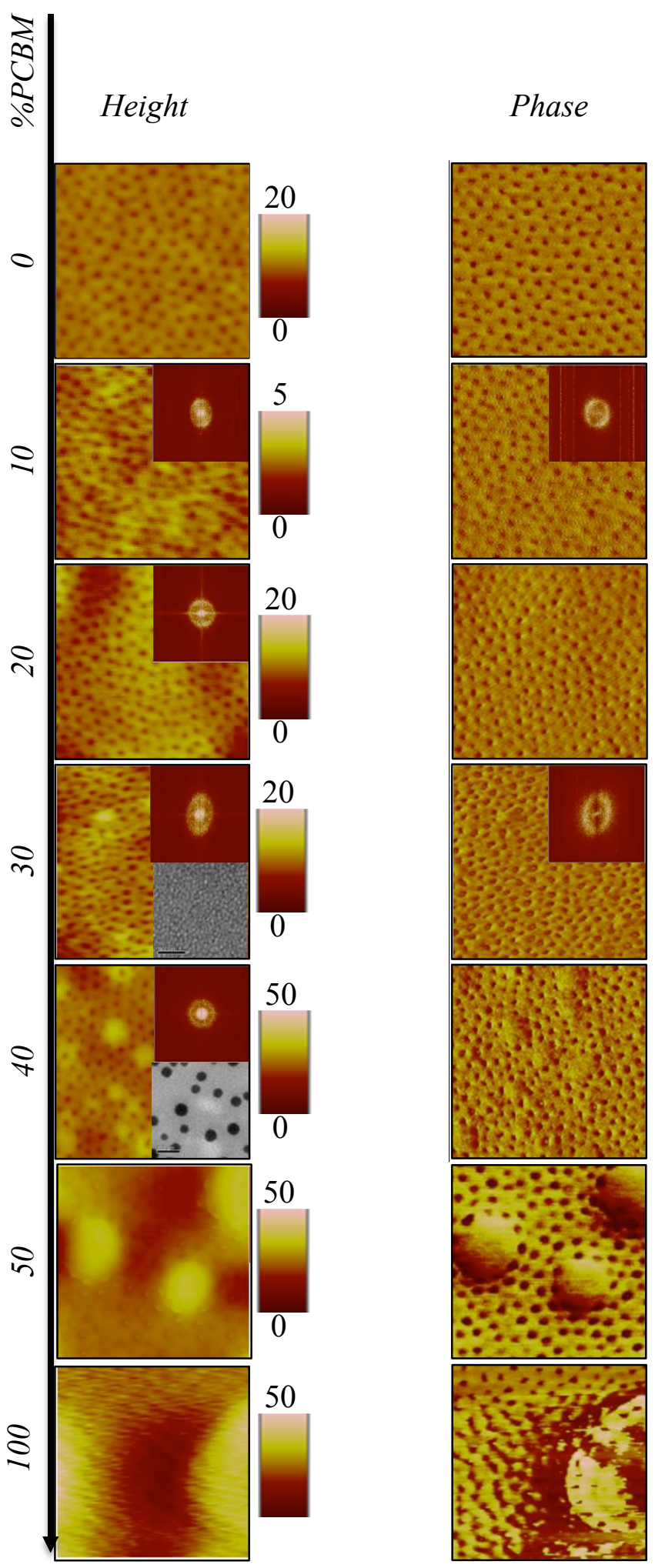

(a) 

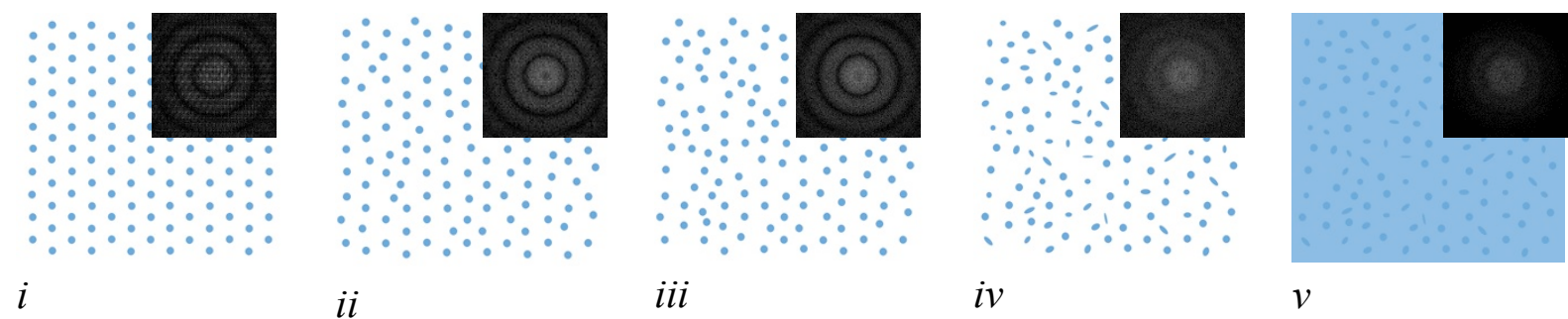

$i v$

$v$

(b)

Figure 1. (a) $500 \mathrm{~nm} \times 500 \mathrm{~nm}$ AFM scan for films cast from toluene with different level of PCBM loadings as marked at the left side of the composite image. Left and right columns represent height and phase images respectively. FFT's are shown as insets at the top right corners of the corresponding images. TEM images for pristine film, as well as for $30 \%$ and $40 \%$ PCBM loaded films are shown as lower right insets of height images. Scales of TEM images are $0.2 \mu \mathrm{m}$. (b) Simulated vertically oriented cylinders and FFT of the different topographical structures of cylinders shown: (i) perfectly hexagonal, (ii) long range ordered, (iii) short range order, (iv) short range order with variable cylinder size, and (v) short range order with variable cylinder size and reduced contrast between a cylinder and the matrix.

and PEO (surface energy $\left.43 \mathrm{~mJ} / \mathrm{m}^{2}\right)^{30}$ have very close surface energy. AFM and Fast Fourier Transforms (FFT) of the images show that the PEO cylindrical domains remain distinct and the PCBM remains dispersed up to 30 mass \% of the polymer in the films. Single broad ring shaped FFT may be due to one or more of the following reasons (see Figure 1b): lack of long range hexagonal packing of the cylinders, shape of the cylinders, or contrast between the matrix and a cylinder, it is worth noting that the ring width shown in Figure 1b-i is related to the diameter of the cylinders; small diameters results in broad and widely spaced concentric rings.

There is no relative enhancement or decrease in the AFM phase contrast between the PEO cylinders and the matrix PS with addition of PCBM, suggesting that up to this level of PCBM loading, the PCBM is uniformly dispersed in the PEO and the PS blocks. Further increase in PCBM loading results in PCBM aggregates appearing as blobs in the AFM images on the top of the film. However, AFM phase images show that even at these high PCBM loadings, the cylindrical structure of the PEO domains of the block copolymer is not destroyed. Thus it seems that the excess PCBM is simply excluded from the well-ordered PS- $b$-PEO structure above a high loading level, rather than lead to a disruption of the structure. The high level of dispersion of PCBM in the BCP films is also confirmed by TEM images, shown as select insets of Figure 1 for corresponding AFM height images. However, it appears that PCBM has no preferential segregation tendency to any particular block as loading of high electron density PCBM does not increase the contrast for any of the blocks. 
TEM images also confirm that the PCBM molecules aggregates when the PCBM loading is at $40 \%$ and higher relative to block copolymer weight. GISAXS measurements examine the internal structures of the films to complement the AFM images that are sensitive to the surface of the films. GISAXS is sensitive to the block copolymer domain, inter-block copolymer domain spacing and long-range order of the PCBM filled PEO cylinders. Average value of line cut is obtained from the region shown by the square box for Figure 2a. GISAXS and its line cuts depict that PEO cylinders having short-range order are present in the films as only first order diffraction is observed. However, from 10\% up to even 50\% PCBM loading level, the block copolymer structure was not destroyed as shown in Figures $2 \mathrm{~b}$ and 2c.

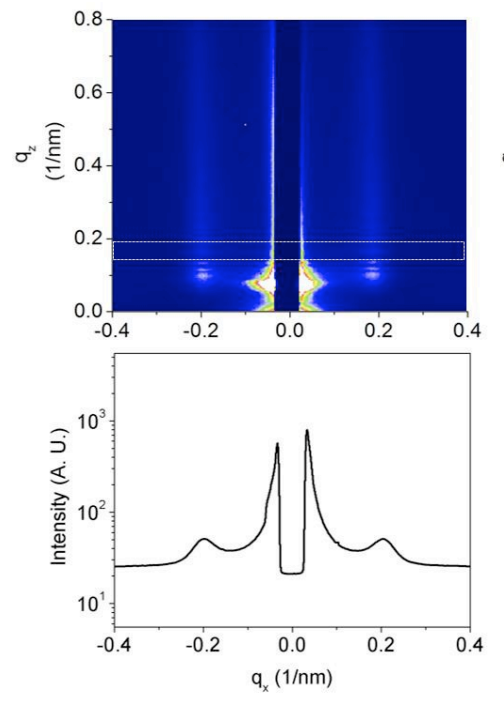

(a)
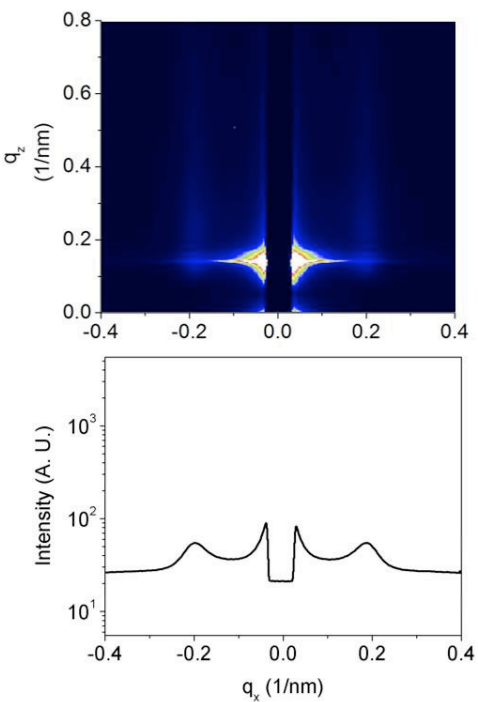

(b)

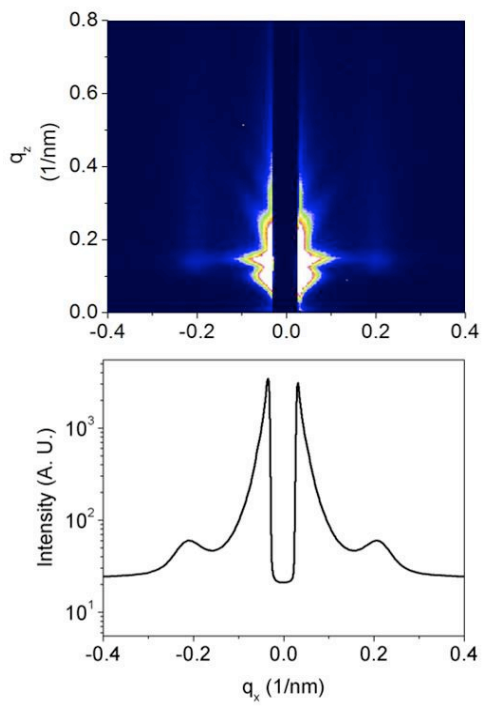

(c)

Figure 2. GISAXS images and line cuts for PS- $b$-PEO:PCBM mixture films spun cast from toluene. Line cut profiles is shown for these films with (a) No PCBM, (b) 10\% PCBM and (c) $50 \%$ PCBM. The horizontal box marked in (a) is the line cut region.

Subsequently we performed GIWAXS on the samples, which is sensitive to a much smaller scale of crystallization, notably PCBM and PEO crystallization. An out of plane 1D intensity vs. $q_{z}$ line cut is shown in Figure 3 along with GIWAXS images. First in Figure 3a we present scattering from PCBM, drop cast from 0.5\% PCBM in dichlorobenzene as a reference. PCBM remains amorphous after casting and forms a diffuse ring of scattering. The block copolymer critical angle is $0.12^{\circ}$, while PCBM and substrate critical angles are around $0.15^{\circ}$ and $0.21^{\circ}$, respectively. The result presented in Figure $3 \mathrm{~b}$ is at the critical angle of the polymer. Under this condition we observe no signature ring of PCBM crystallization in the presence of the PS- $b$-PEO, nor any signature of crystallization of PEO block of the block copolymer. Next, we conducted experiments around the critical angle of PCBM, but below the critical angle of the substrate (Figure 3c). In this case scattering comes from the full thickness of the polymer 
and PCBM composite film. In this instance, we do observe the isotropic PCBM crystallization signature ring, which is clearly demonstrated in the out of plane line cut. Since scattering at $0.12^{\circ}$ does not show any signal coming from PCBM but by further increase in incident angle to the critical angle of PCBM, we see the characteristic PCBM crystallization ring, it can be deduced that PCBM is not included in the film, rather it is deposited at the substrate interface.
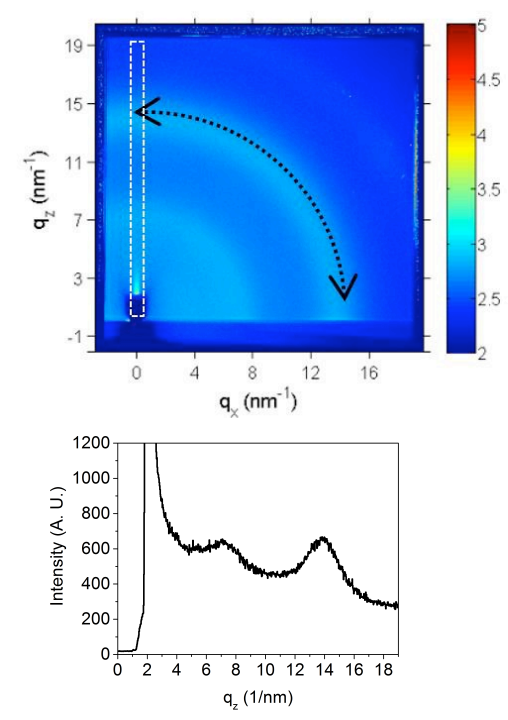

(a)
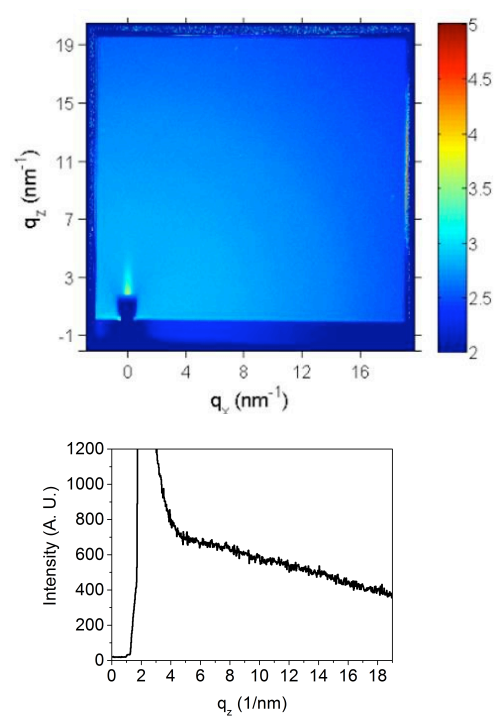

(b)
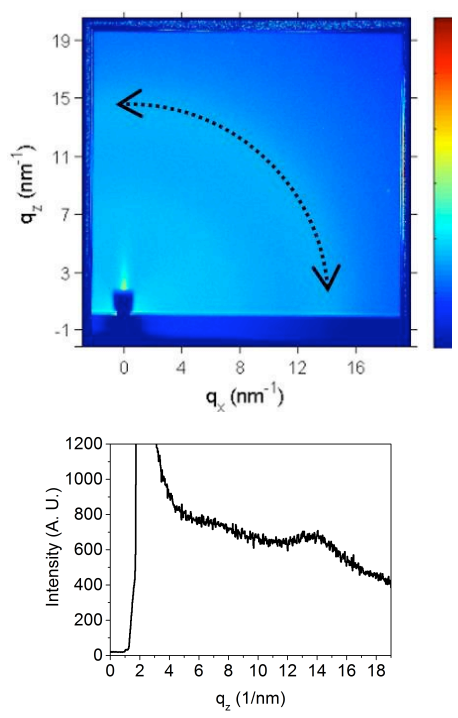

(c)

Figure 3. GIWAXS images for (a) drop cast PCBM, (b) 10:3 PS- $b$-PEO:PCBM films cast from toluene scanned at the critical angle of polymer and (c) at the critical angle of PCBM. The outof-plane line cut position is shown in Figure (a) as a dashed white rectangle. The arrow marks in Figure (a) and (c) indicate the PCBM halo. By curve fitting with a Voigt function of OriginPro software shows that PCBM Characteristic peak is at around $q_{z}$ value of $14 \mathrm{~nm}^{-1}$.

From AFM and TEM images shown in Figure 1 we can deduce that this situation persists until the PCBM loading reaches 30\%, after which it forms aggregates. Even though PCBM has slightly lower surface energy $\left(38.2 \mathrm{~mJ} / \mathrm{m}^{2}\right)^{31}$ as compared to that of PS and PEO, segregation of PCBM to the bottom of the film is due to the rapid adsorption dynamics of the small molecule PCBM from the block copolymer-PCBM solution as compared to the macromolecular block copolymer to the substrate interface. We address agglomerate formation above $30 \%$ loading further in the text.

Figure 4 shows a compendium of information relating to the effect of increasing concentration of PCBM on the nanoscale morphology of the PS- $b$-PEO block copolymer. Plotted are cylinder diameter, center to center distance between cylinders, film thickness (ascast), and RMS roughness. We note that the cylinder diameter as well as center-to-center distance between the cylinders did not increase due to addition of PCBM into the block 
copolymer. It can inferred from the earlier finding that PCBM does not get incorporated into the film at high loading above $30 \%$ by weight. Rather it is deposited at the substrate interface, as confirmed by GIWAXS. This finding is further corroborated by the increase in film thickness with the increase in PCBM loading. The RMS roughness of the film increases slightly due to increase in PCBM loading till 30\% of PCBM loading. After that value, the film roughness increases at a faster rate due to aggregation of PCBM.

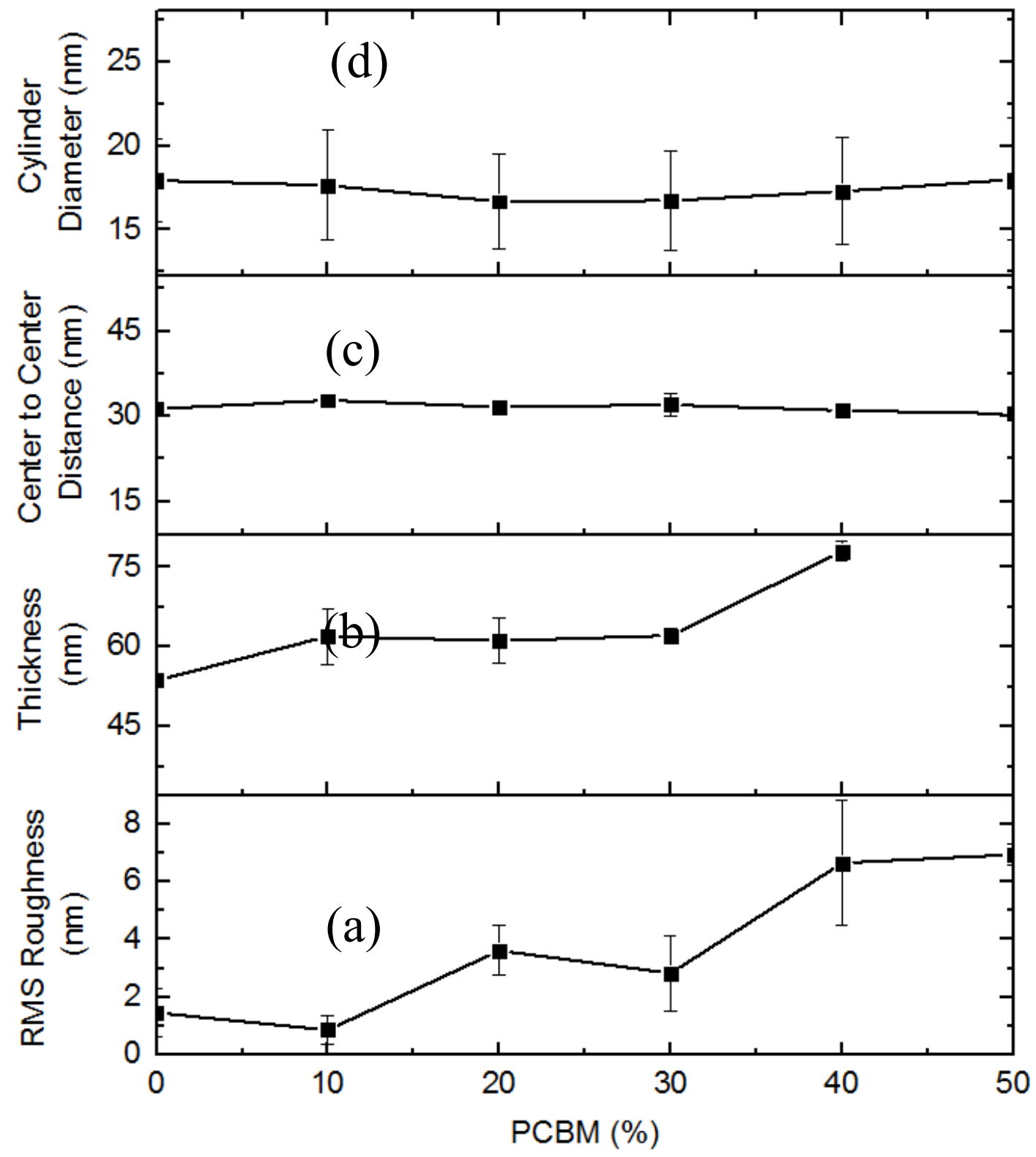

Figure 4. Analysis of PS- $b$-PEO films cast from toluene at different level of PCBM loading. (a) RMS roughness of the top surface of films by AFM, (b) Thickness of the films by AFM scratch test, (c) Average center-to-center distances of cylinders from GISAXS first order peak position, and (d) Cylinder diameters were obtained by AFM phase images of films 


\section{Mixed Solvent Cast Ordered Nanofilled BCP Film Morphologies}

The above results on PS-b-PEO film with PCBM added to casting toluene show that the PCBM did not get incorporated within the PS-b-PEO vertically oriented cylinder structure with any notable amount, even though their concentration in solution relative to block copolymer was up to $50 \%$ by weight. We adapt the route of casting these films from binary solvent mixtures as a versatile route owing to easy tunability of casting solvent mixture properties. However, these include a number of important solvent attributes such as relative solubility of the individual block copolymer PS and PEO blocks and the PCBM in the individual solvents as the primary factor. Brabec et al. ${ }^{38}$ have determined the solubility of PCBM in Toluene, Methanol, Ethanol and DCB to be $15.6,<0.1,<0.1$ and $42.1 \mathrm{mg} / \mathrm{mL}$ respectively . Methanol and Ethanol are bad solvents for PCBM and hence, any solvent combination with relative excess concentration of Methanol and Ethanol will lead to precipitation/agglomeration. Specific simulation or experimental studies are required to determine boundaries. However, in our case particle loading is very low and hence, we did not observe precipitation or agglomeration. Additionally, other factor can be important, that include solvent's evaporation rate. If the two solvent evaporation rates are vastly different, one of the solvent may be retained in the film longer, allowing for interesting ordering dynamics. Furthermore, the preferential interaction of the solvent's wettability on the substrate surface can influence the overall film wettability and possibly even the block orientation on the substrate, although it is

Table 1. Hansen solubility parameters

\begin{tabular}{lccc}
\cline { 2 - 3 } o-Dichlorobenzene (DCB) & 44 & 6.3 & 3.3 \\
Ethanol $^{44}$ & 19.2 & 8.8 & 19.4 \\
Methanol $^{44}$ & 15.8 & 12.3 & 22.3 \\
Toluene $^{44}$ & 15.1 & 1.4 & 2.0 \\
Polystyrene (Polystyrene LG, BASF) $^{45}$ & 18.0 & 5.8 & 4.3 \\
Poly(ethylene oxide) $^{46}$ & 21.3 & 3.0 & 9.4 \\
PCBM $^{47}$ & 17.3 & 6.8 & 6.6
\end{tabular}

believe this is a top-down solvent evaporation driven phenomena. Finally, the choice of binary solvents controls the solvent-solvent miscibility. Casting from immiscible binary solvent can be quite different from miscible ones.

We examine only the primary factor, i.e polymer-solvent miscibility of PS- $b$-PEO films with PCBM cast from dual solvent mixtures with the idea that Hansen solubility parameters can 
be tuned in order to predict the dissolution of each polymer component in such solvents. The basic idea that governs Hansen solubility is that the total cohesion energy is the sum of cohesion energies due to dispersion, polar, and hydrogen bonding interactions. ${ }^{32}$ This leads a correlation between total interaction parameter $\delta_{T}$ to the constituent component interaction parameters, ? ? 2=? ? 2+? 2+? ? 2, where $\delta_{D}, \delta_{P}$, and $\delta_{H}$ are dispersion, polar and hydrogen bonding components. For mixed solvents, the volumetric weight average of each component can be assigned as the corresponding values of mixed solvents. This gives us an effective tool to control the interaction parameter values of a mixed solvent. Interaction parameters of the solvents and polymers used in this research are summarized in Table 1.

In this study we present results on films cast from mixed solvents of $3: 1(\mathrm{w} / \mathrm{w})$ ratio DCB:ethanol, toluene:ethanol and toluene:methanol. We chose a 3:1 ratio because with further increase of the methanol ratio in the toluene-methanol mixture the block copolymer solution becomes turbid because of the onset of precipitation. We used this ratio constant for all other solvent mixtures for fair comparison. Other factors which can be important during spin cast process are summarized in Table 2.

Table 2. Boiling point, viscosity and vapor pressure data of the solvents used in this work

\begin{tabular}{lll}
\hline Boiling Point & & Viscosity \\
$\left({ }^{\circ 3} \mathrm{C}\right)$ & at $20^{\circ} \mathrm{C}(\mu$ Pa.s $)$ & at $20^{\circ} \mathrm{C}(\mathrm{kPa})$ \\
\hline 180.00 & 1450.8 & 0.15 \\
78.29 & 1216.4 & 7.57 \\
64.60 & 593.8 & 16.41 \\
110.63 & 603.6 & 3.75
\end{tabular}

The vapor pressure of a mixed solvent ideal solvents under equilibrium in a closed chamber can be determined by Raoult's law. However, during the spin casting process, the more volatile component of the mixed solvent evaporates at a faster rate. In general it can be said that addition of volatile component results in fast drying. From the viscosity and vapor pressure data it can be deduced that a mixture of toluene and ethanol has higher viscosity and the resulting solvent evaporates at a faster rate as compared to that of pure toluene. So we can expect thicker films from this mixed solvent as compared to films cast from only toluene. Similar reasoning is valid for other solvent compositions. In fact film thicknesses, cast from different solvents and solvent mixtures corroborates this hypothesis that solution viscosity is a parameter that controls film thickness. 
We first consider pure PS- $b$-PEO block copolymer films without any added PCBM, but only cast from different solvent mixtures. Block copolymer concentrations in different solvents are adjusted in this category in order to obtain film thicknesses around $50 \mathrm{~nm}$. In order to achieve expected thickness, the following PS- $b$-PEO concentrations (weight percent) are required: $0.9 \%$ for toluene, $1 \%$ for DCB:ethanol $=3: 1 \quad(\mathrm{w} / \mathrm{w}), 0.75 \%$ for toluene :methanol=3:1 (w/w), $0.63 \%$ for toluene:ethanol $=3: 1$.

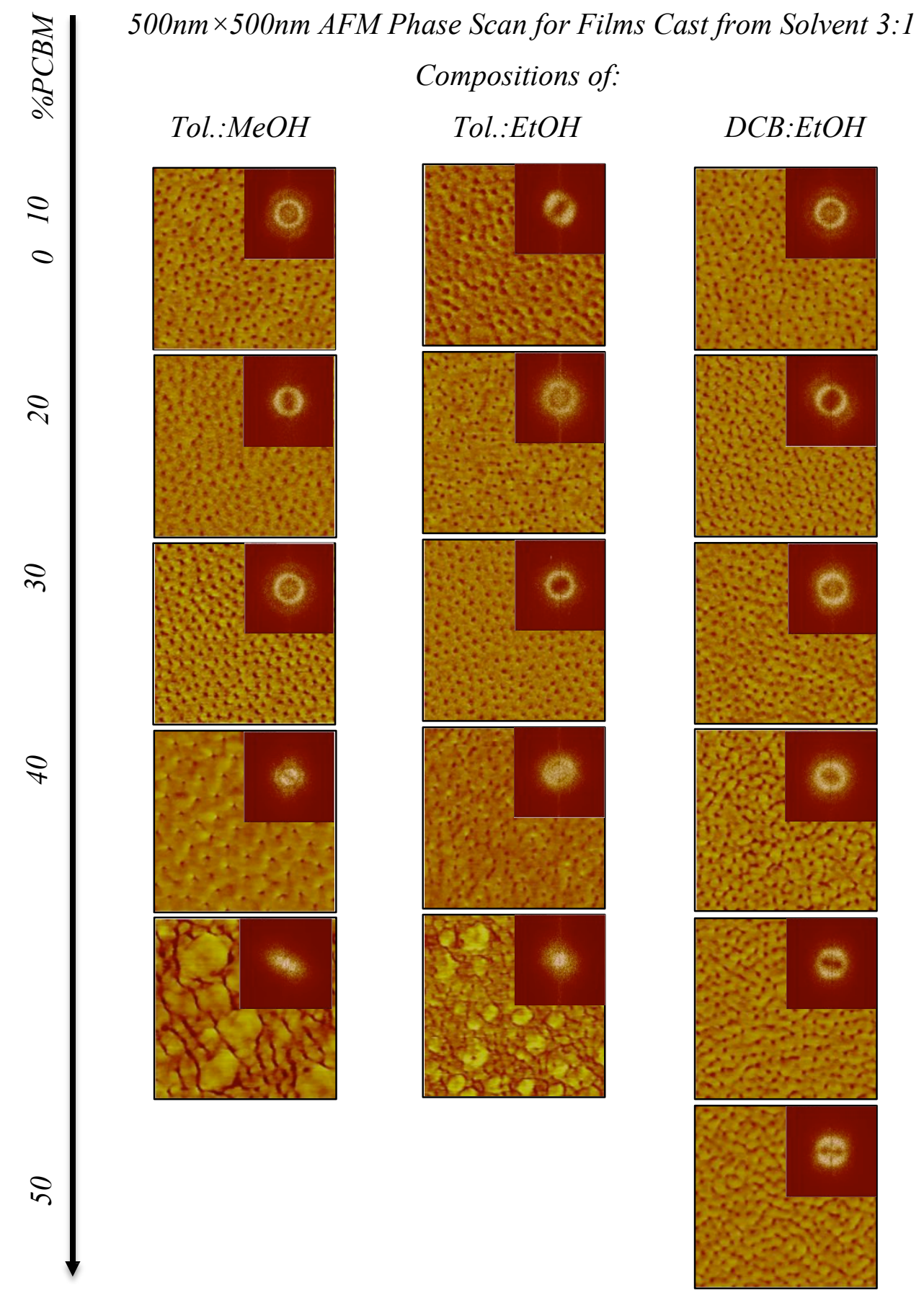


Figure 5. AFM of PS-b-PEO:PCBM (wetting) films cast from binary solvent mixtures. AFM phase images at different levels of PCBM loadings were cast from mixed solvents. PCBM loading levels are presented at the left of the composite image. Corresponding solvent mixtures at 3:1 ratio are marked at the top of each column. FFT's are presented as inserts of the corresponding images.
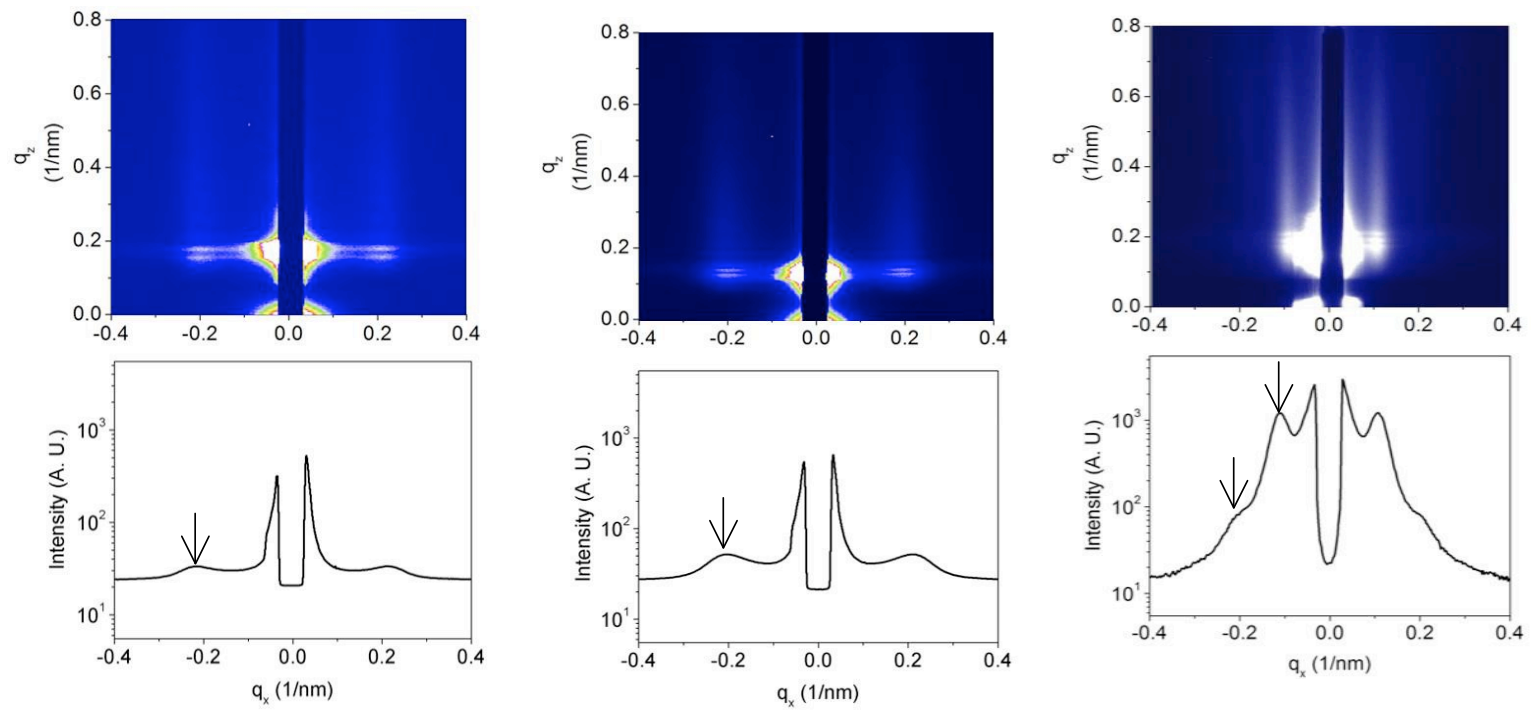

(a)

(b)

(c)

Figure 6. GISAXS images (top) and line cuts (bottom) for films spin cast from toluene. (a) PS- $b$-PEO films cast from o-dichlorobenzene and ethanol mixtures, (b) PS- $b$-PEO and 50\% PCBM films cast from o-dichlorobenzene and ethanol mixtures, (c) PS- $b$-PEO and $30 \%$ PCBM films cast from toluene and methanol mixtures. Peak positions are shown by arrow marks.

We observed that DCB-methanol solvent combinations results in partially dewetted films. Hence, we prepared spin-cast films from the next higher alcohol, ethanol, which has a higher viscosity and slower evaporation rate due to a higher boiling point, as a co-solvent of DCB. Alcohols such as ethanol and methanol are good solvent for PEO domains but nonsolvent for PS domain. DCB,on the other hand, is a good solvent for PS, so during spin casting alcohol preferentially migrate towards PEO domain and hence, drives the hydrophobic PCBM molecules in the PS domain. Results for uniform films are achieved from DCB-ethanol mixture are shown in Figures 5 and 7 comprising of AFM images and their respective analysis. The general observation is that the film remained wetting even up to $50 \%$ of PCBM loading relative to the PS-b-PEO block copolymer weight. FFT's of the corresponding images are an indication of the goodness of hexagonal packing of PEO cylinders in the matrix. However, we observe that neither the PS nor the PEO phase swells in the cast ordered film. Therefore in this case, like pure toluene cast films, we conclude that PCBM is segregated at the substrate interface with increasing concentration of PCBM. This is apparently due to slower evaporation rate of ethanol compared to toluene (discussed later), 
enabling preferential segregation of polar PCBM towards the substrate while the solvents evaporate. Next, toluene-ethanol and toluene-methanol mixed solvents were used for casting. Both cases yield uniform films with all vertical PEO cylinders. At 30\% PCBM loading, a dramatic increase of PS domain size is observed and a slight decrease in PEO domain size is observed which is in accordance with phenomena observed by Winey et al. ${ }^{39}$, when cast from toluene-methanol mixed solvent, and notably, we observe that PCBM does not form agglomerates even up to the $30 \% \mathrm{wt}$ fraction loading. The decrease is relatively small ( observed at high $\sim 30 \%$ loading of PCBM) possibly due to the small size of PCBM, and lack of correlated structure, such as observed in homopolymers. Further, the added PCBM leads to an overall film thickness increase where they are significantly accommodated. Since the solution properties of this casting mixture are somewhat special (turbid and spin-casting conditions) as described later, we leave this interesting aspect of domain shrinkage for future studies.

We performed GISAXS experiments on the films cast from mixed solvents to confirm their internal structure. GISAXS on DCB-ethanol cast films with different PCBM loading, yield results with first order peaks and not much change in domain spacing. In Figure 6, we present the contrast between the film with highest PCBM loading incorporated within the film leading to domain spacing changes, i.e. 30\% PCBM loading, as well as that of neat PS-b-PEO, and 50\% PCBM wt. fraction in solution that is not incorporated in any significant amount in the film for. comparison. The optimal choice of toluene-methanol mixture is evident and clearly important. The GISAXS of the 30\% PCBM loaded films cast from toluene-methanol mixture are consistent with topographic finding by AFM of domain swelling, and we observe a second order peak, indicating longer range ordering of cylinder domains (see Figure 6c).

Figure 7 summarizes the results obtained from AFM and GISAXS experiments. As shown in the figure, relatively low change in PEO cylinder diameters indicates that PCBM is barely incorporated into this particular phase. Center-to-center distance of PEO cylinders also remain unchanged for films cast from DCB-ethanol and toluene-ethanol mixed solvents. When cast from toluene-methanol solvent pair, a notable increase in center-to-center distance was obtained from both GISAXS and AFM results for the 30\% PCBM loaded PS-b-PEO film, which indicates that majority PCBM is integrated into the PS phase in the cast film, discussed in detail below. In cases of toluene-ethanol and DCB-ethanol casting, the film thicknesses progressively increase with PCBM loading due to adsorption of PCBM at the polymer-substrate interfaces not due to incorporation of PCBM in polymer film. Since PCBM has excellent solubility in DCB we observe low film RMS roughness values when the casting solvent is DCB:ethanol=3:1 as PCBM is expectedly more uniformly distributed at the polymer-substrate interface.

One of the reasons we chose the reported ratio and choice of toluene-methanol solvents was because the PS-b-PEO with PCBM solution was on the border between a turbid and clear solution as observed optically. A lightly turbid solution may indicate that the $\mathrm{BCP}$ forms a 
suspension, rather than an immiscible solution, in which case it may completely precipitate out from the solution. Nevertheless, there were some spin coating challenges with this solution. Even a 30\% PCBM loaded solution in a toluene:methanol $=3: 1$ solvent mixture precipitated within few seconds after drop casting on silicon and no cylinder morphology could be observed at that point after spin casting. Hence, spin coating was started within $\sim 1$ second after drop casting. Rapid co-precipitation of polymer and PCBM during spin casting entraps PCBM into the polymer film before it can phase segregate at the substrate. ${ }^{35}$ Consequently, PCBM is preferentially segregated into the PS phase due to difference in interactions of PCBM nanoparticles and the constituents of the $\mathrm{BCP}$ in the mixed solvent. At room temperature a toluene-methanol solvent has a Hansen 


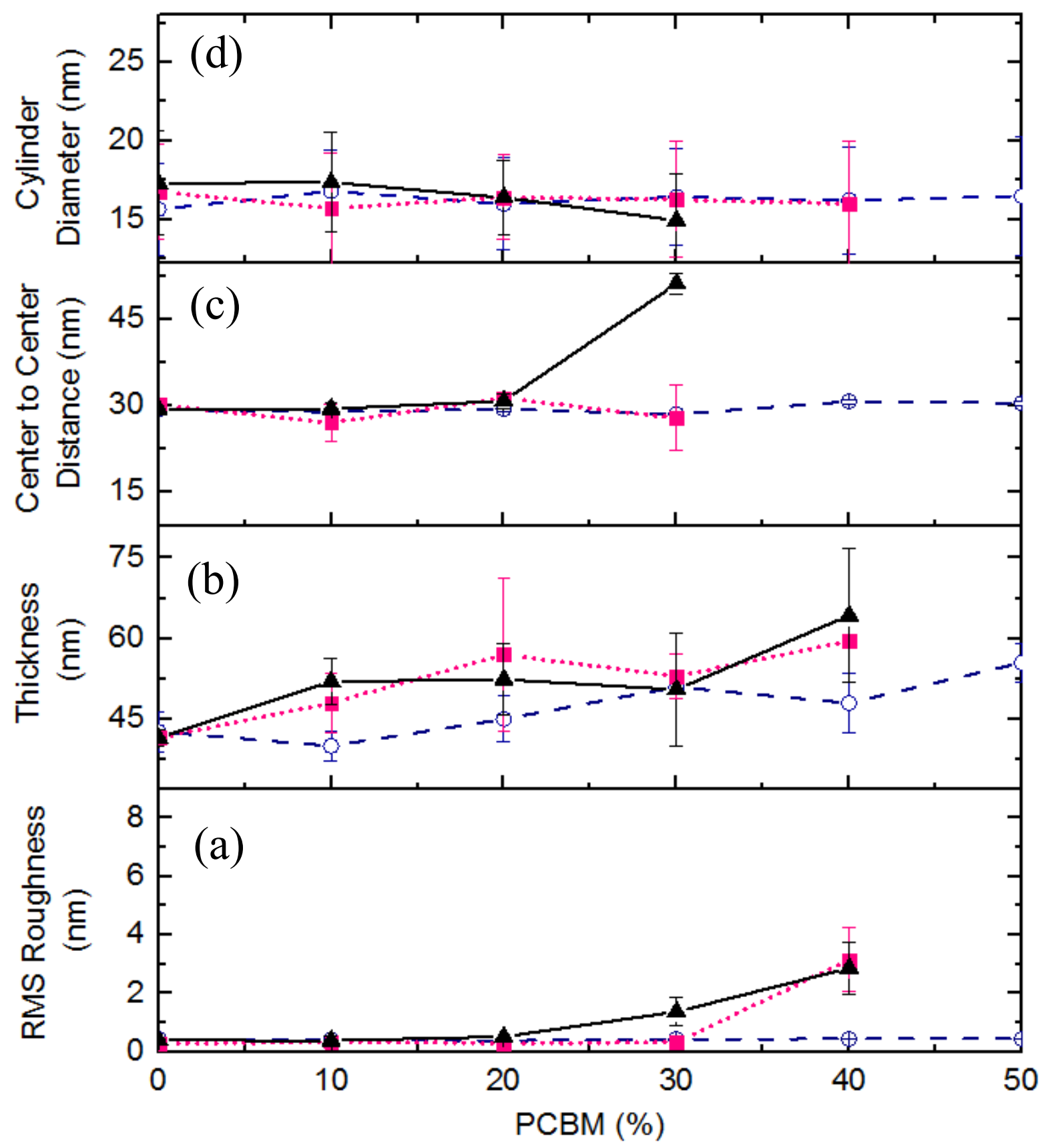

Figure 7. Analysis of PS- $b$-PEO films cast from mixed solvents at different level of PCBM loading. Symbols $\mathrm{O}, \boldsymbol{\mathbf { }}$, and $\boldsymbol{\Delta}$ indicates films cast from DCB-ethanol, toluene-ethanol, and toluene-methanol mixed solvents. (a) RMS roughness of the top surface of films by AFM, (b) Thickness of the films by AFM scratch test, (c) Average center-to-center distances of cylinders from GISAXS first order peak position, and (d) Cylinder diameter of the films by power spectral density of AFM phase image of films. 
solubility parameter of $7.4 \mathrm{MPa}^{1 / 2}$ due to hydrogen bonding. This lies in-between the values of PEO $\left(\delta_{H}\right.$ for $\left.\mathrm{PEO}=9.4 \mathrm{MPa}^{1 / 2}\right)$ and PS $\left(\delta_{H}\right.$ for $\left.\mathrm{PS}=4.3 \mathrm{MPa} .^{1 / 2}\right)$. We hypothesize that as the film dries, the solvent gets cooler because the evaporating solution takes latent heat of vaporization from the substrate and solution. Consequently, the value of hydrogen bonding in methanol, present in PEO phase, increases. But the polymer hydrogen bonding remains similar. Now that there is more deviations, both in terms of polar, as well as hydrogen bonding between the methanol rich PEO phase and PCBM $\left(\delta_{P=6.8} \mathrm{MPa}^{1 / 2}\right.$ and $\left.\delta_{H}=6.6 \mathrm{MPa}^{1 / 2}\right)$, the PCBM nanoparticles are driven into the PS phase, which has relatively similar values of $\delta_{P}$ and $\delta_{H}$ (for PS $\delta_{P}=5.8 \mathrm{MPa} .{ }^{1 / 2}$ and $\delta_{H}=4.3 \mathrm{MPa} .{ }^{1 / 2}$ ). Moreover, incorporation of PCBM into PS phase is also enthalpically favorable because of the favorable interaction between PS phenyl group and aromatic "bucky ball" nature of PCBM. PCBM migration into PS is further facilitated by the fact that the small molecule methanol acts as plasticizer that enables facile migration of PCBM. ${ }^{32}$ The other factor that may contributes to the dramatic increase in PS domain size is due to the fact that a nanoparticle increases the free volume of the PS domain as proposed by Mackay et $a l^{36}$ However, this is a less likely, since it applies only when there is almost no solvent in the film to approach the melt state of the film, at which point the PCBM mobility is greatly diminished. Particle aggregation beyond $30 \%(\mathrm{w} / \mathrm{w})$ loading (in mixed as well as pure solvent system) seems to be kinetic phenomena. As determined by Brabec et al. ${ }^{38}$, Methanol and Ethanol are bad solvent for PCBM. While, Toluene and DCB can be loaded with higher quantities of PCBM (as mentioned previously). It is less likely that these aggregates are formed in solution state because concentration of polymer and particles in solution is very low $(<1 \%)$. While spin casting, concentration of PCBM increases and hence, polymer-PCBM-solvent mixture reaches solubility limit faster in highly loaded samples (assuming same evaporation rate). So during later stage, the limited solubility of PCBM in polymer becomes relevant and aggregates start to form.

In the schematic of Figure 8 we illustrate the effect of mixed toluene-methanol solvent on relative PS domain swelling of PS- $b$-PEO block copolymer. This is due to preferential segregation of PCBM into PS, so that the mixed solvent approach must modify the conformal entropy of the PS matrix. As previously mentioned, this casting solvent mixture and casting conditions are somewhat special.

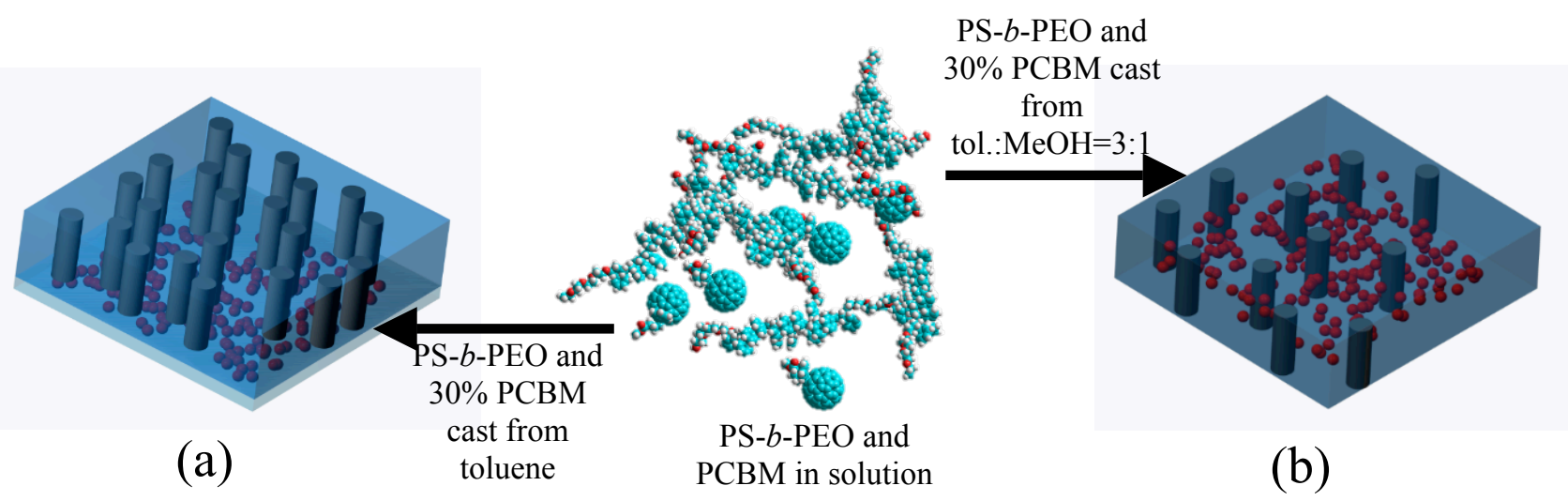


Figure 8. Schematic of film cast from (a) toluene and (b) toluene-methanol mixed solvent. In case of films cast from toluene, PCBM is deposited at the substrate interface. However, when cast from the mixed solvent, PCBM dispersed into PS phase of PS- $b$-PEO block copolymer.

\section{Conclusions}

In this paper, functional nanoparticles were introduced in amphiphilic block copolymer films to understand the correlation between nanoparticle loading versus changes in block copolymer film morphology and orientation. Specifically, the dispersion and segregation of PCBM nanoparticles into the different domains of PS-b-PEO was studied with the purpose of understanding the correlation to changes in the solvent cast film morphology. The effect of casting solvent-polymer mixture comprised of both single and mixed solvents was studied. PCBM incorporation in the PS block using solvent mixtures, optimized for preferential segregation of PCBM into PS matrix based on Hansen solubility parameters was considered. We demonstrate that controlled solvent casting from lightly turbid solutions by rapidly spincasting before precipitation on substrate occurs can incorporate PCBM selectively in PS-b-PEO. Such incorporation of nanoparticles is due to the appropriate choice of a solvent pair and their mixing ratio, so that PCBM is kinetically entrapped into the PS-b-PEO film which otherwise has the natural tendency to segregate to the substrate-polymer interface. Subsequently, such entrapped PCBM is segregated into PS phase due to change of Hansen solubility parameters and plasticizing effect of the resulting solvents during spin casting process as well as its chemical affinity towards PS phase. This strategy allowed us to incorporate up to $30 \%$ by weight of PCBM relative to PS-b-PEO, while maintaining its vertical morphology, important to nanotechnology applications for modifying film properties for applications such as polymer based solar cells and dielectrics for energy storage. Our mixed-solvent strategy may be generalized to other PCBM-BCP mixtures that phase separate or segregate or precipitate when cast from a single solvent.

\section{Acknowledgements}

The AFM characterization of morphology of thin films for high-energy capacitor design was supported by AFOSR contract No. FA9550-12-1-0306. Analysis of the homogeneity and dispersion of the PCBM within the polymer film for suitability for dielectrics and future glass transition studies was enabled by support from the W.M. Keck Foundation. The GISAXS scattering aspects of the work was funded by Department of Energy, Basic Energy Sciences via grant DE-FG02-10ER4779. CHESS at Cornell University is supported by the NSF \& NIH/NIGMS via NSF award DMR-1332208. 


\section{References:}

(1) Bates, F. S.; Fredrickson, G. H. Block Copolymer Thermodynamics: Theory and Experiment. Annu. Rev. Phys. Chem. 1990, 41, 525-557.

(2) Singh, G.; Yager, K. G.; Berry, B.; Kim, H.-C.; Karim, A. Dynamic Thermal FieldInduced Gradient Soft-Shear for Highly Oriented Block Copolymer Thin Films. ACS Nano 2012, 6, 10335-10342.

(3) Fredrickson, G. H.; Bates, F. S. Dynamics of Block Copolymers: Theory and Experiment. Annu. Rev. Mater. Sci. 1996, 26, 501-550.

(4) Bates, F. S. Polymer-Polymer Phase Behavior. Science (80-. ). 1991, 251, 898-905.

(5) Singh, G.; Yager, K. G.; Smilgies, D.-M.; Kulkarni, M. M.; Bucknall, D. G.; Karim, A. Tuning Molecular Relaxation for Vertical Orientation in Cylindrical Block Copolymer Films via Sharp Dynamic Zone Annealing. Macromolecules 2012, 45, 7107-7117.

(6) Kim, S.; Briber, R. M.; Karim, A.; Jones, R. L.; Kim, H.-C. Environment-Controlled Spin Coating to Rapidly Orient Microdomains in Thin Block Copolymer Films. Macromolecules 2007, 40, 4102-41055.

(7) Segalman, R. A. Patterning with Block Copolymer Thin Films. Mater. Sci. Eng. R 2005, $48,191-226$.

(8) Tang, C.; Wu, W.; Smilgies, D.-M.; Matyjaszewski, K.; Kowalewski, T. Robust Control of Microdomain Orientation in Thin Films of Block Copolymers by Zone Casting. J. Am. Chem. Soc. 2011, 133, 11802-11809.

(9) Darling, S. B. Block Copolymers for Photovoltaics. Energy Environ. Sci. 2009, 2, 12661273.

(10) Aissou, K.; Kogelschatz, M.; Baron, T.; Gentile, P. Self-Assembled Block Polymer Templates as High Resolution Lithographic Masks. Surf. Sci. 2007, 601, 2611-2614.

(11) Aissou, K.; Baron, T.; Kogelschatz, M.; Hertog, M. Den; Rouviere, J. L.; Hartmann, J.M.; Pelissier, B. Fabrication of Well-Organized and Densely Packed Si Nanopillars Containing Sige Nanodots by Using Block Copolymer Templates. Chem. Mater. 2008, 20, 6183-6188.

(12) Choi, D.-G.; Jeong, J.-R.; Kwon, K.-Y.; Jung, H.-T.; Shin, S.-C.; Yang, S.-M. Magnetic Nanodot Arrays Patterned by Selective Ion Etching Using Block Copolymer Templates. Nanotechnology 2004, 15, 970-974. 
(13) Darling, S. B.; Yufa, N. a.; Cisse, a. L.; Bader, S. D.; Sibener, S. J. Self-Organization of FePt Nanoparticles on Photochemically Modified Diblock Copolymer Templates. Adv. Mater. 2005, 17, 2446-2450.

(14) Guarini, K. W.; Black, C. T.; Zhang, Y.; Kim, H.; Sikorski, E. M.; Babich, I. V. Process Integration of Self-Assembled Polymer Templates into Silicon Nanofabrication. J. Vac. Sci. Technol. B Microelectron. Nanom. Struct. 2002, 20, 2788.

(15) Mansky, P.; Liu, Y.; Huang, E.; Russell, T. P.; Hawker, C. Controlling Polymer-Surface Interactions with Random Copolymer Brushes. Science (80-. ). 1997, 275, 1458-1460.

(16) Shin, K.; Leach, K. A.; Goldbach, J. T.; Kim, D. H.; Jho, J. Y.; Tuominen, M.; Hawker, C. J.; Russell, T. P. A Simple Route to Metal Nanodots and Nanoporous Metal Films. Nano Lett. 2002, 2, 933-936.

(17) Thurn-Albrecht, T.; Schotter, J.; Kästle, G. a; Emley, N.; Shibauchi, T.; Krusin-Elbaum, L.; Guarini, K.; Black, C. T.; Tuominen, M. T.; Russell, T. P. Ultrahigh-Density Nanowire Arrays Grown in Self-Assembled Diblock Copolymer Templates. Science 2000, 290, 2126-2129.

(18) Haryono, A.; Binder, W. H. Controlled Arrangement of Nanoparticle Arrays in BlockCopolymer Domains. Small 2006, 2, 600-611.

(19) Aissou, K.; Fleury, G.; Pecastaings, G.; Alnasser, T.; Mornet, S.; Goglio, G.; Hadziioannou, G. Hexagonal-to-Cubic Phase Transformation in Composite Thin Films Induced by FePt Nanoparticles Located at PS/PEO Interfaces. Langmuir 2011, 27, 1448114488 .

(20) Chai, J.; Buriak, J. M. Using Cylindrical Domains of Block Copolymers To SelfAssemble and Align Metallic Nanowires. ACS Nano 2008, 2, 489-501.

(21) Guo, Y.; Harirchian-Saei, S.; Izumi, C. M. S.; Moffitt, M. G. Block Copolymer Mimetic Self-Assembly of Inorganic Nanoparticles. ACS Nano 2011, 5, 3309-3318.

(22) Kao, J.; Bai, P.; Chuang, V. P.; Jiang, Z.; Ercius, P.; Xu, T. Nanoparticle Assemblies in Thin Films of Supramolecular Nanocomposites. Nano Lett. 2012, 12, 2610-2618.

(23) Lin, Y.; Böker, A.; He, J.; Sill, K.; Xiang, H.; Abetz, C.; Li, X.; Wang, J.; Emrick, T.; Long, S.; et al. Self-Directed Self-Assembly of Nanoparticle/Copolymer Mixtures. Nature 2005, 343, 55-59.

(24) Niu, S.; Saraf, R. F. Selective Assembly of Nanoparticles on Block Copolymer by Surface Modification. Nanotechnology 2007, 18, 125607(1)-125607(4).

(25) Sarkar, B.; Alexandridis, P. Self-Assembled Block Copolymer-Nanoparticle Hybrids: Interplay between Enthalpy and Entropy. Langmuir 2012, 28, 15975-15986. 
(26) Warren, S. C.; Messina, L. C.; Slaughter, L. S.; Kamperman, M.; Zhou, Q.; Gruner, S. M.; DiSalvo, F. J.; Wiesner, U. Ordered Mesoporous Materials from Metal NanoparticleBlock Copolymer Self-Assembly. Science (80-. ). 2008, 320, 1748-1752.

(27) Dang, M. T.; Hirsch, L.; Wantz, G. P3HT:PCBM, Best Seller in Polymer Photovoltaic Research. Adv. Mater. 2011, 23, 3597-3602.

(28) Hiemenz, P. C.; Lodge, T. P. Polymer Chemistry; Second Edi.; CRC Press: Boca Raton, 2007; p. 224.

(29) Scheer, H.-C.; Schulz, H.; Hoffmann, T.; Torres, C. M. S. Handbook of Thin Film Materials; Nalwa, H. S., E., Ed.; First Edit.; Academic Press: San Diego, California, 2002; p. 47.

(30) Van Oss, C. J.; Chaudhury, M. K.; Good, R. J. Monopolar Surfaces. Adv. Colloid Interface Sci. 1987, 28, 35-64.

(31) Watts, B.; Belcher, W. J.; Thomsen, L.; Ade, H.; Dastoor, P. C. A Quantitative Study of PCBM Diffusion during Annealing of P3HT:PCBM Blend Films. Macromolecules 2009, 42, 8392-8397.

(32) Hansen Solubility Parameters; Hansen, C. M., Ed.; Second Edi.; Taylor \& Francis Group, LLC: Boca Raton, 2007; pp. 7, 19, 104.

(33) CRC Handbook of Chemistry and Physics; David R. Lide, Ed.; 87th Editi.; CRC Taylor \& Francis: Boca Raton, 2006; pp. 3-150 to 3-486, 6-177 to 6-181.

(34) Liley, P. E.; Thomson, G. H.; Friend, D. G.; Daubert, T. E.; Buck, E. Perry's Chemical Engineers' Handbook; Perry, R. H.; Green, D. W., Eds.; Seventh ed.; McGraw-Hill, 1997; pp. 2-68 to $2-74$.

(35) Du, F.; Fischer, J. E.; Winey, K. I. Coagulation Method for Preparing Single-Walled Carbon Nanotube/poly(methyl Methacrylate) Composites and Their Modulus, Electrical Conductivity, and Thermal Stability. J. Polym. Sci. Part B Polym. Phys. 2003, 41, 33333338 .

(36) Mackay, M. E.; Dao, T. T.; Tuteja, A.; Ho, D. L.; van Horn, B.; Kim, H.-C.; Hawker, C. J. Nanoscale Effects Leading to Non-Einstein-like Decrease in Viscosity. Nat. Mater. 2003, 2, 762-766.

(37) Zhang, R.; Singh, G.; Dang, A.; Dai, L.; Bockstaller, M. R.; Bulent, A.; Satija, S.; Karim, A. Nanoparticle-Driven Orientation Transition and Soft-Shear Alignment in Diblock Copolymer Films via Dynamic Thermal Gradient Field Macromol. Rapid Commun. 2013, $34,1642-1647$.

(38) Machui, F.; Langner, S.; Zhu, X.; Abbott, S.; Brabec, C. Determination Of The 
P3HT:PCBM Solubility Parameters Via A Binary Solvent Gradient Method: Impact Of Solubility On The Photovoltaic Performance. Solar Energy Materials and Solar Cells 2012, 100, 138-146.

(39) Winey, K.; Thomas, E.; Fetters, L. Swelling Of Lamellar Diblock Copolymer By Homopolymer: Influences Of Homopolymer Concentration And Molecular Weight. Macromolecules 1991, 24, 6182-6188. 